\title{
Hyperbolic semi-adequate links
}

David Futer, Efstratia Kalfagianni and Jessica S. Purcell

We provide a diagrammatic criterion for semi-adequate links to be hyperbolic. We also give a conjectural description of the satellite structures of semi-adequate links. One application of our result is that the closures of sufficiently complicated positive braids are hyperbolic links.

1 Introduction

1.1 Volume bounds and relations

1.2 Satellite semi-adequate links

1.3 Organization

2 Background and tools

2.2 Polyhedral toolbox

3 Tori, annuli, and squares 1006

4 Diagrammatically incompressible annuli

5 Diagrammatically compressible annuli

6 Seifert fibered link complements

References 


\section{Introduction}

The problem of determining the geometric structure of a link complement from a link diagram is both important and hard. A related, similarly difficult, problem asks for relations between geometric and diagrammatic invariants of a link. The purpose of this paper is to discuss these problems for the class of semi-adequate links. We give diagrammatic criteria for such links to be hyperbolic, and state a conjecture about their satellite structures. Semi-adequate links form a very broad class of links that first appeared in the study of Jones-type invariants [23, 29], and have since been studied considerably from the point of view of both quantum topology and geometric topology; see [14] and references therein.

In [13], we developed a framework for establishing relations between geometric and combinatorial link invariants. In particular, to a semi-adequate link diagram we associate a certain graph (state graph) and a surface spanned by the link, and construct a certain ideal polyhedral decomposition of the surface complement. We use normal surface theory to show that combinatorial properties of the state graph dictate the structure of the JSJdecomposition of the surface complement, and encode geometric information of the link complement. For instance we show that, for hyperbolic semiadequate links, graph theoretic invariants coarsely determine the volume of the link [13] and the geometric types of certain essential surfaces in the link complement [15]. The machinery of [13] lends itself naturally to the study of essential surfaces in link complements via normal surface theory.

In this paper, we focus on essential tori and annuli in link complements, and give a diagrammatic criterion that rules them out, implying the link is hyperbolic. For links that fail this criterion, we give a conjectural description of the satellite structures. Our results place several known classes of hyperbolic links under a common umbrella and lead to new constructions of such links.

To state our results, we need to briefly explain the related terminology; we give precise definitions in Section 2. For every semi-adequate link diagram there is a corresponding state graph $\mathbb{G}$. The edges of $\mathbb{G}$ are in one-to-one correspondence with the crossings of the link diagram. One way to obtain 2 -edge loops in $\mathbb{G}$ is from crossings in the same twist region of the link diagram, where edges of $\mathbb{G}$ corresponding to crossings of that twist region are parallel between two vertices of $\mathbb{G}$. Our result concerns link diagrams for which all the 2-edge loops of $\mathbb{G}$ are obtained this way. 

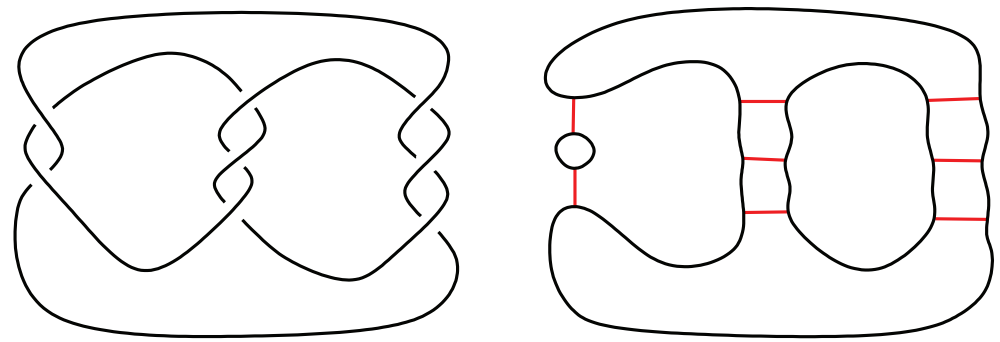

Figure 1: Left: the $(-2,3,3)$ pretzel knot. Right: the graph of the all $-A$ resolution contains $2-$ edge loops that do not belong to a single twist region.

Theorem 1.1. Suppose that $D(K)$ is a connected, prime, semi-adequate diagram with at least two twist regions, such that for each 2-edge loop in the corresponding state graph, the edges belong to the same twist region. Then the link $K$ depicted by this diagram is hyperbolic.

Figure 1 shows an example of a connected, prime, semi-adequate diagram that doesn't satisfy the 2-edge loop hypothesis of Theorem 1.1. This diagram represents the $(-2,3,3)$ pretzel knot, which is known to be equivalent to the $(3,4)$ torus knot, hence is not hyperbolic. This shows that the 2 -edge loop condition is necessary for Theorem 1.1. The class of links with semi-adequate diagrams that don't satisfy the 2-edge loop condition is quite large, and contains plenty of hyperbolic knots (e.g. the $(-2,3,7)$ pretzel) and satellite knots (see Example 1.5).

Theorem 1.1 is reminiscent of a result of Menasco, which states that any link admitting a connected, prime, alternating diagram with at least two twist regions is hyperbolic [24]. In fact, the hypotheses of Theorem 1.1 apply in particular to prime, alternating, twist-reduced diagrams. In this setting, the statement of the theorem reduces to Menasco's result.

In addition, Theorem 1.1 generalizes Menasco's result to large classes of non-alternating links. For a warm-up example, consider the non-alternating pretzel link diagram of the form $P\left(a_{1}, \ldots, a_{r}, b_{1}, \ldots, b_{s}\right)$ that has $r$ vertical bands containing $a_{1}, \ldots, a_{r}$ positive crossings, and $s$ vertical bands containing $b_{1}, \ldots, b_{s}$ negative crossings. See Figure 1 for the example of $P(-2,3,3)$, and [23, Figures 1 and 2] for the general case. If $r, s \geq 3$ and $a_{i}, b_{i} \geq 3$, for all $i$, then the diagrams satisfy the hypothesis of Theorem 1.1. More generally, one may obtain families of non-alternating Montesinos or arborescent links by imposing similar restrictions on the rational tangles involved. Note that 
a classification of hyperbolic arborescent links is already known by the work of Bonahon and Siebenmann [6, 9].

For a sample class of non-alternating links whose hyperbolicity can be established for the first time using Theorem 1.1, consider the family of positive or negative closed braids with at least 3 crossings per twist region.

Corollary 1.2. Let $B_{n}$ be the braid group on $n$ strands, with $n \geq 3$, and let $\sigma_{1}, \ldots, \sigma_{n-1}$ be the elementary braid generators. Let $b=\sigma_{i_{1}}^{r_{1}} \sigma_{i_{2}}^{r_{2}} \cdots \sigma_{i_{k}}^{r_{k}}$ be a braid in $B_{n}$. Suppose that either $r_{j} \geq 3$ for all $j$, or else $r_{j} \leq-3$ for all $j$. Suppose moreover that the braid closure $D_{b}$ of $b$ is a prime diagram. Then the link $K$ depicted by this diagram is hyperbolic.

Several other applications of Theorem 1.1 are given by Giambrone [17]. For instance, he proves that for a sufficiently complicated braid $b$, the plat closure of $b$ is hyperbolic.

The problem of determining the geometric structures of link complements from link diagrams has been studied considerably in the literature. In addition to the work of Menasco on alternating links, Bonahon and Siebenmann [6] classified the geometric types of arborescent links and showed that with some explicitly described exceptions, these links are hyperbolic. See also Futer and Guéritaud [9] for a direct proof. Adams showed that augmented alternating links are hyperbolic [1]. He also showed that toroidally alternating links are either composite, torus knots, or hyperbolic [2], although determining which of the three occurs is difficult. More recently, Futer and Purcell showed that prime link diagrams in which each twist region has at least six crossings represent either $(2, q)$ torus links or hyperbolic links [16]. Purcell investigated the geometric structures of certain families of links with multiply twisted regions $[27,28]$. For similar results on other classes of knots and links, we refer the reader to Adams' survey paper [3].

As a corollary of Theorem 1.1, we conclude that the primality of a link can be easily read off from a diagram.

Corollary 1.3. Let $D(K)$ be a connected, semi-adequate diagram without nugatory crossings. Suppose that for each 2-edge loop in the corresponding state graph, the edges belong to the same twist region of $D(K)$. Then $K$ is a prime link if and only if $D(K)$ is prime.

Corollary 1.3 is reminiscent of some prior results for more restricted link families. For instance, it generalizes a theorem of Menasco: if $D(K)$ is a connected alternating diagram, then $K$ is prime if and only if $D(K)$ is prime 
[24]. Similarly, Ozawa proved that if $D(K)$ is a connected positive diagram, then $K$ is prime if and only if $D(K)$ is prime [25].

In fact, the hypothesis on 2-edge loops in Corollary 1.3 should be unnecessary. It is conjectured that a connected, prime, semi-adequate diagram must always represent a prime link (see [13, Problem 10.6] and [26]). Corollary 1.3 gives a partial solution to this conjecture.

Finally, we note that a connected, semi-adequate diagram always represents a non-split link. See Thistlethwaite [29, Corollary 3.2] for the original proof, relying on properties of link polynomial invariants, and Ozawa [26, Theorem 2.15] for an alternate, geometric proof. Thus for the rest of the paper, we will assume our diagrams are connected, and hence the link is non-split.

\subsection{Volume bounds and relations}

The machinery of [13] allows for connections between geometric invariants of a link complement, combinatorial properties of its diagram, and stable coefficients of its colored Jones polynomials. The class of links of Theorem 1.1 is particularly well suited for such applications. For instance, [13, Corollary 9.4] relates the hyperbolic volume of these links to the Euler characteristic of the corresponding reduced state graph. (See Definition 2.2 for the terminology and notation.)

Corollary 1.4. Suppose that $K$ is a link with prime, semi-adequate diagram $D(K)$ as in the statement of Theorem 1.1. Then

$$
\operatorname{vol}\left(S^{3} \backslash K\right) \geq-v_{8} \chi\left(\mathbb{G}^{\prime}\right)
$$

where $v_{8}=3.6638 \cdots$ is the volume of a regular ideal octahedron and $\mathbb{G}^{\prime}$ is $\mathbb{G}_{A}^{\prime}$ or $\mathbb{G}_{B}^{\prime}$ according to whether $D(K)$ is $A$-adequate or $B$-adequate.

This corollary has applications in two directions. First, coupled with the upper volume bounds given by Lackenby, Agol, and Thurston, [22], and combined with additional work of Giambrone [17], it leads to two-sided bounds on $\operatorname{vol}\left(S^{3} \backslash K\right)$ in terms of the twist number of a semi-adequate diagram. This extends a result of Lackenby [22] and results of the authors [10-12] to new link families. Second, it leads to two-sided bounds on $\operatorname{vol}\left(S^{3} \backslash K\right)$ in terms of stable coefficients of the colored Jones polynomials of $K$, as predicted by the Coarse Volume Conjecture [13, Question 10.13]. Details are given in Giambrone [17]. 


\subsection{Satellite semi-adequate links}

As we mentioned above, there is a conjectural strengthening of Corollary 1.3, which removes the hypothesis on 2-edge loops. Formulating the right conjectural strengthening of Theorem 1.1 requires some care, as there are many non-hyperbolic semi-adequate links. For example, it is well-known that all torus links are semi-adequate. Similarly, all planar cables of a semi-adequate diagram are semi-adequate [7]. There are also many semi-adequate satellites, as the following construction illustrates.

Example 1.5. Recall that a satellite link is constructed from a companion knot $J \subset S^{3}$, a pattern link $K^{\prime} \subset D^{2} \times S^{1}$, and an embedding $f: D^{2} \times S^{1} \rightarrow$ $N(J)$. The image $K=f\left(K^{\prime}\right)$ will be a non-trivial satellite whenever $J$ is nontrivial, and $K^{\prime}$ is not the core of the solid torus or contained in a ball in the solid torus. The whole construction can be performed diagrammatically: given a diagram $D(J) \subset \mathbb{R}^{2}$, and a diagram $D\left(K^{\prime}\right) \subset[0,1] \times S^{1}$, the blackboard framing of $D(J)$ specifies a way to immerse the annulus $I \times S^{1}$ into $\mathbb{R}^{2}$, which gives a diagram $D(K)$. See Figure 2 .

Suppose $D(J) \subset \mathbb{R}^{2}$ and $D\left(K^{\prime}\right) \subset I \times S^{1}$ are both $A$-adequate diagrams. Consider the graph $H_{A}\left(K^{\prime}\right)$ coming from $K^{\prime}$ (see Definitions 2.1 and 2.2). Suppose that there is a rectangle $R=I \times I \subset I \times S^{1}$, such that if we remove the state circles and segments of $H_{A}$ that lie entirely in $R$, what remains is $n \geq 1$ copies of the core curve $\{*\} \times S^{1}$.

Now, suppose that we use the blackboard framing of $D(J)$ to immerse the annulus $I \times S^{1}$ into $\mathbb{R}^{2}$, so that the image rectangle $f(R)$ lies in a crossingfree region of $D(J)$. Outside the image rectangle $f(R)$, the graph $H_{A}$ of the resulting diagram $D(K)$ will look identical to the graph of the $n$-fold planar cable of $J$, which is known to be $A$-adequate. Inside $f(R)$, the diagram is $A$-adequate because $D\left(K^{\prime}\right)$ is $A$-adequate. See Figure 2 for an example, which produces the Whitehead double of the trefoil.

Conjecture 1.6. Suppose $D(K)$ is a semi-adequate diagram of a satellite link $K$. Then $D(K)$ or its mirror image can be obtained using the construction of Example 1.5. In particular, the satellite torus must be visible in $D(K)$ as the regular neighborhood of an immersed annulus.

\subsection{Organization}

The paper is organized as follows. In Section 2, we summarize definitions and the main tools from [13] that are needed in this paper. To a semi-adequate 

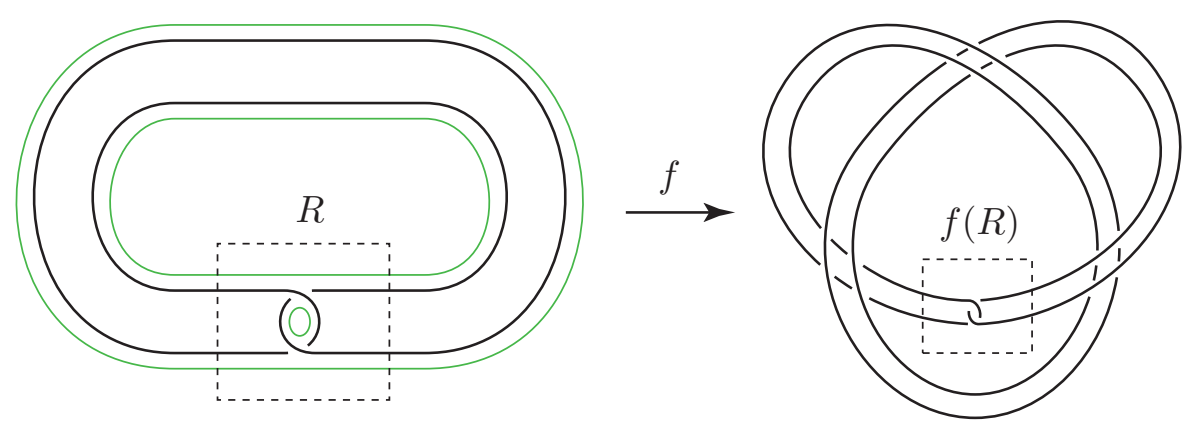

Figure 2: Left: a diagram $D\left(K^{\prime}\right)$ in an annulus. State circles of the all- $A$ resolution are shown in green. After removing state circles in a rectangle $R$, what remains is two parallel copies of the core. Right: an embedding of this annulus into a regular neighborhood of the trefoil produces an $A$-adequate diagram of a Whitehead double.

diagram $D(K)$, we associate a state surface $S_{A}$ that is essential in the complement of $K$. Its complement $M_{A}=S^{3} \backslash \backslash S_{A}$ admits a checkerboard ideal polyhedral decomposition (see $\S 2.2$ ). The intersection of an essential torus in $S^{3} \backslash K$ with $M_{A}$ is a collection of essential annuli that can be studied using normal surface theory with respect to the polyhedral decomposition. We discuss this in Section 3, where we also show that the resulting essential annuli in $M_{A}$ fall into two types: diagrammatically compressible and diagrammatically incompressible (see Definition 3.2).

In Sections 4 and 5, we analyze the two types of annuli and conclude that under the hypotheses of Theorem 1.1, neither of the two types arise as part of an essential torus. We note that our analysis of diagrammatically incompressible annuli in Section 4 does not require the hypotheses of Theorem 1.1. It leads to a classification of such annuli in complements of all semi-adequate links. We expect that these results will have further applications, including in approaching Conjecture 1.6. In Section 5, where we study diagrammatically compressible annuli, a key ingredient is a classification of essential product disks in polyhedra from [13]. The main result in Sections 4 and 5 is Theorem 5.10: if a diagram $D(K)$ is as in the statement of Theorem 1.1, then $S^{3} \backslash K$ is atoroidal.

In Section 6, we complete the proof of Theorem 1.1 and Corollaries 1.2 and 1.3. Our approach in this section is to rule out the possibility that $S^{3} \backslash K$ might be Seifert fibered, using Gromov norm estimates and Turaev surface methods. 


\section{Background and tools}

We begin this section by recalling definitions of semi-adequate knots and related terms. We also review some constructions from [13] that we will be using throughout the paper.

\subsection{Definitions}

For any crossing of a link diagram $D:=D(K)$, there are two resolutions, called the $A$-resolution and $B$-resolution of the crossing, as in Figure 3.

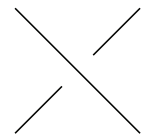

Figure 3: $A$ - and $B$-resolutions of a crossing.

A choice of $A$ - or $B$-resolution for each crossing of $D$ is called a Kauffman state [20]. The result of applying a state to $D$ a collection of circles disjointly embedded in the projection plane. These are called state circles.

Throughout this paper, we will be concerned only with the all- $A$ and all- $B$ states, which correspond to making a uniform choice of $A$ or $B$ at all crossings.

Definition 2.1. Given a diagram $D(K)$ and the all- $A$ state of $D$, we construct a trivalent graph $H_{A}$ as follows. For each resolved crossing of $D$, add an edge between resulting state circles, shown dashed in Figure 3. Every edge of $H_{A}$ either belongs to a state circle of the all- $A$ resolution, or comes from a crossing. The latter edges are called segments.

Similarly, we define a trivalent graph $H_{B}$, whose edges consist of state circles and segments of the $B$-resolution. Observe that the original link diagram $D(K)$ can be reconstructed from the graph $H_{A}$ or $H_{B}$.

Definition 2.2. The state graphs $\mathbb{G}_{A}$ and $\mathbb{G}_{B}$ are obtained from $H_{A}$ and $H_{B}$, respectively, by collapsing each state circle to a vertex. Removing redundant edges between vertices, we obtain the reduced state graphs $\mathbb{G}_{A}^{\prime}$ and $\mathbb{G}_{B}^{\prime}$.

Following Lickorish and Thistlethwaite [23, 29], a diagram $D$ is said to be $A$-adequate if every edge of $\mathbb{G}_{A}$ has its endpoints on distinct vertices. Similarly, one can define $B$-adequate diagrams using $\mathbb{G}_{B}$. A link diagram that is either $A$-adequate or $B$-adequate is called semi-adequate. 
A link $K$ will be called $A$-adequate ( $B$-adequate) if it admits an $A$ adequate ( $B$-adequate) diagram. A link that is either $A$-adequate or $B$ adequate is called semi-adequate.

Definition 2.3. A link diagram $D$ is called prime if any closed curve in the projection plane that meets the diagram transversely exactly twice bounds a region of the diagram with no crossings.

A twist region of $D$ is a collection of bigons in $D$ that are adjacent end to end, such that there are no additional adjacent bigons on either end. An example of such a twist region is shown at the top of Figure 4. A single crossing adjacent to no bigons is also a twist region. We require twist regions to be alternating, for if $D$ contains a bigon that is not alternating, then a Reidemeister move removes both crossings without altering the rest of the diagram. The number of distinct twist regions in a diagram is defined to be the twist number of that diagram. Note that if $D$ has exactly one twist region, it is a closed 2 -braid; i.e. the standard diagram of a $(2, q)$ torus link.

To understand the statement of Theorem 1.1, we need to explain the hypothesis that for each 2-edge loop in the state graph, the edges belong to the same twist region of $D(K)$. To make this precise, we need the following definition.

Definition 2.4. Suppose $R$ is a twist region of a link diagram $D$ such that $R$ contains $c_{R}>1$ crossings. Consider the all $-A$ and all $-B$ resolutions applied to $R$. One of the state graphs, say $\mathbb{G}_{B}$, will inherit $c_{R}-1$ vertices from the $c_{R}-1$ bigons contained in $R$. We say that this is the long resolution of $R$. The other graph, say $\mathbb{G}_{A}$, contains $c_{R}$ parallel edges only one of which survives in $\mathbb{G}_{A}^{\prime}$. This is the short resolution of $R$. See Figure 4 .

Throughout the paper, we will be concerned with semi-adequate diagrams where the 2-edge loops in the corresponding state graph come from short resolutions of twist regions.

Definition 2.5. Suppose that the state graph, say $\mathbb{G}_{A}$, contains a 2-edge loop. We say that the two edges of that loop belong to the same twist region $R$ of the diagram if first, the edges come from resolving two crossings in $R$, and second, the resolution of $R$ in $\mathbb{G}_{A}$ is the short one. If every 2-edge loop in $\mathbb{G}_{A}$ has its edges in the same twist region, we say that $\mathbb{G}_{A}$ satisfies the 2-edge loop condition. See Figure 1 for an example that fails the 2-edge loop condition. 


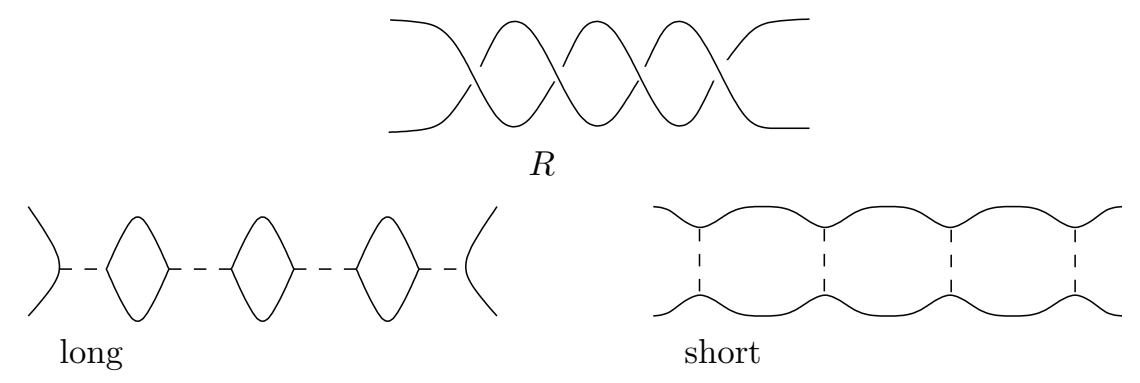

Figure 4: Resolutions of a twist region $R$.

Suppose $\gamma$ is a simple closed curve meeting the diagram $D(K)$ exactly twice in two crossings $x_{1}, x_{2}$. Adjust $\gamma$ in a neighborhood of each crossing, so that after the adjustment $\gamma$ meets the diagram exactly four times, and has a subarc $\gamma_{i}$ in the neighborhood of $x_{i}$, with endpoints on the projection of $K$. Now consider the $A$ - and $B$-resolutions of the two crossings. For each $x_{i}$, exactly one of these resolutions will produce a segment that is parallel to $\gamma_{i}$. When $\gamma$ meets two crossings in a twist region, then the resolution producing the segment parallel to $\gamma_{i}$ is the short resolution of the twist region.
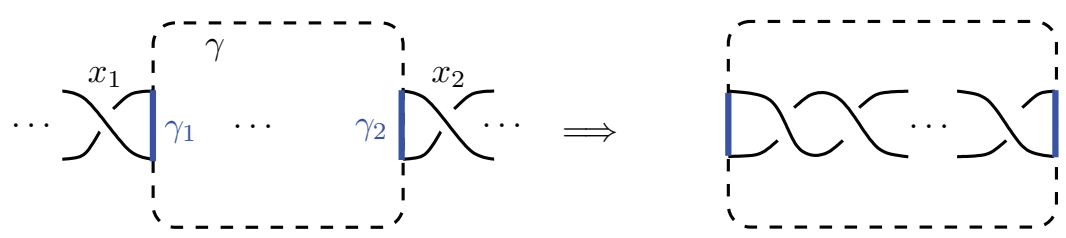

Figure 5: The property of being $A$-twist reduced. Whenever there is a closed curve $\gamma$ meeting the diagram as shown on the left, the crossings $x_{1}$ and $x_{2}$ must belong to the same twist region, as shown on the right. Note that the twist region containing $x_{1}, x_{2}$ can lie on either side of $\gamma$.

Definition 2.6. We say that a diagram is $A$-twist reduced if it satisfies the following property. Suppose $\gamma$ is a simple closed curve $\gamma$ meeting the diagram exactly four times adjacent to two crossings, as above, such that the all- $A$ state produces segments parallel to subarcs of $\gamma$. Then $\gamma$ bounds a subdiagram consisting of a (possibly empty) collection of bigons arranged in a row between the two crossings. See Figure 5. 
The property of being $B$-twist reduced is defined in the same way, with the all- $B$ state replacing the all $-A$ state. Adequate diagrams that are both $A$ - and $B$-twist reduced are twist reduced in the sense of Lackenby [22].

In the sequel, we will consider $A$-adequate diagrams for simplicity. The same results hold for $B$-adequate diagrams, by taking a mirror image.

Lemma 2.7. Let $D(K)$ be an $A$-adequate diagram such that $\mathbb{G}_{A}$ satisfies the 2-edge loop condition. Then $D(K)$ is A-twist reduced.

Proof. Suppose that $\gamma$ is a closed curve as in Definition 2.6, meeting the diagram exactly four times adjacent to two crossings, $x_{1}$ and $x_{2}$. Let $\gamma_{i}$ denote the subarc of $\gamma$ which runs between two points on the diagram and lies in a neighborhood of the crossing $x_{i}$. Suppose that the $A$-resolution of $x_{1}$ and $x_{2}$ produces segments parallel to $\gamma_{1}$ and $\gamma_{2}$. Consider how the state circles of the all $-A$ state intersect the region inside $\gamma$.

First, note that if some state circle runs from $x_{i}$ back to $x_{i}$, this state circle will violate the definition of $A$-adequacy. Therefore, there must be two state circles running from $x_{1}$ to $x_{2}$, and the two edges of $\mathbb{G}_{A}$ corresponding to those crossings give a 2-edge loop. By hypothesis, the edges belong to the same twist region $R$, which means the edges come from resolving two crossings of the twist region $R$ in $\mathbb{G}_{A}$. Then by definition there must be a (possibly empty) collection of bigons between $x_{1}$ and $x_{2}$, as desired.

\subsection{Polyhedral toolbox}

The main technical tool that we use is a decomposition of the link complement into an $I$-bundle over a surface and a collection of ideal polyhedra [13, Chapters 2-4]. In order to make this paper as self-contained as possible, we will review the definitions and constructions from there that are relevant to this paper. We will also recall the statements of some key results that are needed below. On those occasions when we rely on results from [13] that are not restated in this section, we will refer the reader to the exact statement of the result we are using in that monograph. The background we provide here should suffice for checking and absorbing the statements in these cases. The reader need only consult the monograph [13] in order to learn the detailed proofs.

For a reader who is new to this material, we also recommend consulting the survey paper [14] for a quick guide to the key features of the polyhedral decomposition. 
Definition 2.8. A diagram $D(K)$ determines a state surface $S_{A}$, constructed as follows. Each state circle of $H_{A}$ bounds a disk, and the disks associated to all the state circles can be disjointly embedded in the 3-ball below the projection plane. (Note this collection of disks in the lower 3-ball is unique up to isotopy.) Every crossing of $D(K)$ gives a segment of $H_{A}$, which runs between two state circles. We connect the corresponding disks by a half-twisted band, twisted in the direction of the original crossing. The result is a (possibly non-orientable) surface $S_{A}$, whose boundary is $K$.

When $D(K)$ is an $A$-adequate diagram, Ozawa [26] showed that $S_{A}$ is an essential surface in $S^{3} \backslash K$. A different proof is given in [13].

Definition 2.9. Let $M_{A}:=S^{3} \backslash N\left(S_{A}\right)$ denote the complement of an open regular neighborhood of $S_{A}$. When convenient, we will also use the shorthand notation $S^{3} \backslash \backslash S_{A}$ instead of $S^{3} \backslash N\left(S_{A}\right)$. The boundary of $M_{A}$ decomposes into the parabolic locus (the remnants in $M_{A}$ of the boundary tori of $S^{3} \backslash K$ ), and a surface $\widetilde{S_{A}}$ that can be identified as the frontier of $N\left(S_{A}\right)$ in $S^{3} \backslash K$. Note that $\widetilde{S_{A}}$ is a double cover of $S_{A}$, connected if and only if $S_{A}$ is nonorientable.

The main technical tool of [13] that we use is a decomposition of $M_{A}$ into ideal polyhedra. The faces of these polyhedra are checkerboard colored, white and shaded. The white faces of each polyhedron are glued to another polyhedron, while the shaded faces lie on $\widetilde{S_{A}}$. There is exactly one upper polyhedron, which occupies the 3 -ball above the projection plane. There are multiple lower polyhedra, each of which is glued along its white faces to the upper polyhedron only. All the polyhedra are prime, in the sense that a pair of faces share at most one edge.

The precise combinatorics of the ideal polyhedra can be read off from the diagram $D(K)$ and the graph $H_{A}$. (See [13, Chapter 2] and [14, Section 5] for details on how to do this.) Here, we describe the features that are salient for this paper.

The first feature we will need is information about the combinatorics of the lower polyhedra. This information comes from subgraphs of $H_{A}$, or slight modifications of subgraphs, which we call polyhedral regions. Their precise definition is as follows.

Definition 2.10. Suppose $\alpha$ is an arc in the complement of $H_{A}$ with both endpoints on a state circle $C$. Consider the subgraph of $H_{A}$ consisting of $C$ and all state circles and segments which lie on the same side of $C$ as $\alpha$. 

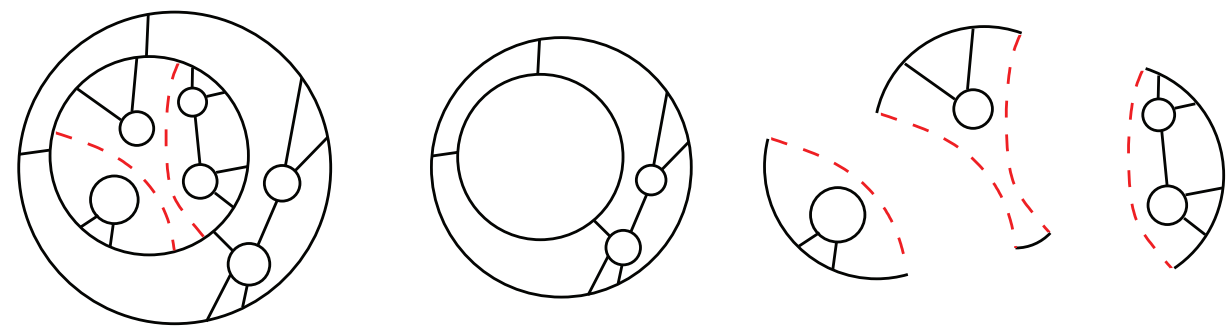

Figure 6: Left: A graph $H_{A}$ with a maximal collection of non-prime arcs (shown in dashed red). Right: This example breaks into four distinct polyhedral regions, as shown.

Note that $\alpha$ cuts the subgraph into two components, one on either side of $\alpha$. If both components contain segments, then we say $\alpha$ is a non-prime arc. A collection of non-prime arcs is maximal if, once we cut along all such arcs and all state circles, the graph decomposes into subgraphs that each contain a segment, and no larger collection of non-prime arcs has this property. Figure 6 , left, shows an example of a graph $H_{A}$ with a maximal collection of non-prime arcs.

Let $\left\{\alpha_{1}, \ldots, \alpha_{n}\right\}$ denote a maximal collection of non-prime arcs. A polyhedral region is a nontrivial region of the complement of the state circles and the $\alpha_{i}$, where by nontrivial we mean the region contains segments. Each lower polyhedron corresponds to precisely one of these polyhedral regions. Note that if $H_{A}$ admits no non-prime arcs, then a polyhedral region is just a region of the complement of the state circles which contains segments. Figure 6 shows an example.

Each white face of the polyhedra corresponds to a nontrivial (i.e. noninnermost disk) complementary region of $H_{A} \cup\left(\cup_{i=1}^{n} \alpha_{i}\right)$. The white faces that belong to a lower polyhedron are glued to corresponding white faces in the unique upper polyhedron.

Associated to each polyhedral region $R$, and a corresponding lower polyhedron $P$, we have a clockwise map. Loosely speaking, the clockwise map $\phi$ gives us a way to associate the lower polyhedron $P$ with a section of the upper polyhedron.

Definition 2.11. The clockwise map $\phi$ is a homeomorphism from the white faces of the upper polyhedron belonging to the polyhedral region $R$ to the white faces of the corresponding lower polyhedron $P$. On each white face, $\phi$ 


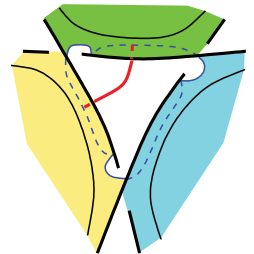

In $S^{3} \backslash K$

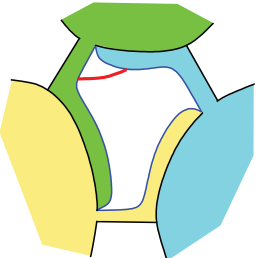

Upper polyhedron

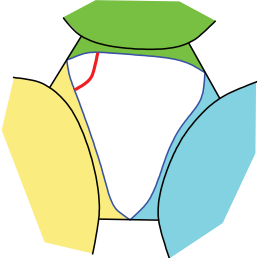

(a) Gluing map

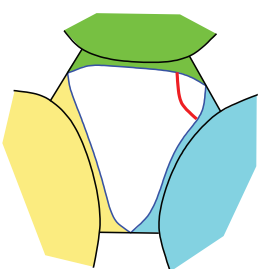

(b) Clockwise map

Figure 7: An edge (in red) in the link complement is shown, along with its position in the upper polyhedron, and images under the gluing and clockwise maps.

is defined by composing the gluing map to a white face of $P$ with a single clockwise rotation in that face.

Figure 7 shows an example of how the clockwise map compares to the gluing map.

There is a way to extend the domain of definition of the clockwise map to normal squares, that is, normal disks with 4 sides. We will use this multiple times in this paper. The following is a restatement of [13, Lemma 4.8].

Lemma 2.12. Let $S$ be a normal square in the upper polyhedron, with arcs $\beta_{V}$ and $\beta_{W}$ in white faces $V, W$ that belong to a polyhedral region $R$. Let $P$ be the lower polyhedron corresponding to $R$, with clockwise map $\phi$. Then there is a normal square $\phi(S) \subset P$, unique up to normal isotopy, which contains white sides $\phi\left(\beta_{V}\right)$ and $\phi\left(\beta_{W}\right)$.

Furthermore, if $S$ is glued along $V$ to a square $T$ in the lower polyhedron, then $\phi(S) \cap V$ will differ from $T \cap V$ by a single clockwise rotation of $V$.

\section{Tori, annuli, and squares}

In this section, we will consider essential tori embedded in the complement of a semi-adequate knot. We will see that the state surface $S_{A}$ cuts these into annuli, and we will consider properties of these annuli. In particular, we will show that the annuli decompose into squares, all of which are either diagrammatically compressible or diagrammatically incompressible. This sets the stage for the next two sections, in which these two cases are analyzed separately. 
Lemma 3.1. Let $D(K)$ be an $A$-adequate diagram, with all-A state surface $S_{A}$. Let $T$ be an essential torus. Then we may isotope $T$ such that $T \backslash \widetilde{S_{A}}$ is an even number of essential annuli, half embedded in $M_{A}$ and half embedded in the $I$-bundle $N\left(S_{A}\right)$.

Furthermore, those essential annuli in $M_{A}$ are cut into normal squares by the white faces of the polyhedral decomposition of $M_{A}$, where each square has two opposite sides on shaded faces and two opposite sides on white faces.

Proof. Isotope $T$ to be transverse to $S_{A}$ and minimize the number of curves of intersection with $S_{A}$. Because $S_{A}$ and $T$ are both essential, this ensures that all intersections $T \cap S_{A}$ are nontrivial simple closed curves on $T$. Hence $T \cap N\left(S_{A}\right)$ and $T \backslash N\left(S_{A}\right)$ consist of annuli, which are essential because $T$ is essential.

Because the annuli in the closure of $T \backslash N\left(S_{A}\right)$ in $M_{A}$ are essential, we can put them into normal form with respect to the polyhedral decomposition of $M_{A}$. This may involve isotopy of the boundary components of the annuli. We may isotope the adjacent annuli in $N\left(S_{A}\right)$ to ensure that when we isotope annuli into normal form, we actually isotope the entire torus.

Let $E \subset T \cap M_{A}$ be an essential annulus in normal form. Since $E$ cannot be contained in a single polyhedron (because it is essential), it must intersect the white faces. We claim that no arc of intersection between $E$ and a white face can be parallel to the boundary of $E$. This is because an outermost such arc would cut off a normal bigon in an ideal polyhedron, and our prime ideal polyhedra do not contain normal bigons [13, Proposition 3.18]. Therefore, every arc of intersection between $E$ and a white face runs across $E$, from one boundary circle to the other. These arcs cut $E$ into normal squares, finishing the lemma.

We will investigate the annuli and squares of Lemma 3.1. The study of these surfaces will naturally break into two cases: whether the squares cut off a single ideal vertex in a white face, or whether each edge in a white face cuts off multiple ideal vertices on both sides. This is encoded in the following definition.

Definition 3.2. Let $S \subset M_{A}$ be a surface in normal form. We say that $S$ is diagrammatically compressible if, in some white face $W$ of the polyhedral decomposition, an arc of $S \cap W$ runs between two adjacent edges of $W$. In other words, $S$ is diagrammatically compressible in $W$ if $S \cap W$ cuts off an ideal vertex of $W$. See Figure 8. Otherwise, if no such white face exists, we call $S$ diagrammatically incompressible. 


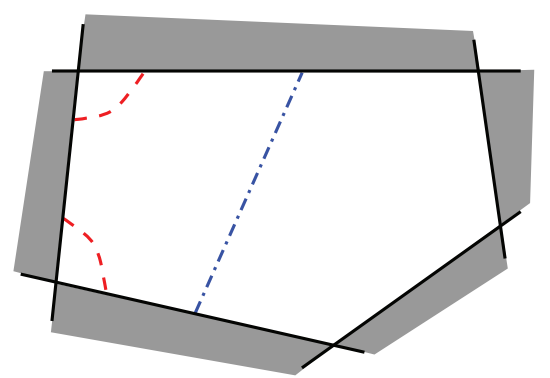

Figure 8: Shown is a white face $W$ of the polyhedral decomposition. Red arcs (dashed) cut off a single ideal vertex of $W$. A surface meeting $W$ in such an arc is diagrammatically compressible. A surface meeting $W$ in the blue arc (dot-dashed) is diagrammatically incompressible, provided its intersections with other white faces also do not cut off a single ideal vertex.

If $S$ is diagrammatically compressible in some white face $W$, it cuts off a disk $U \subset W$ with two sides on shaded faces, one side on $S$, and one side on an ideal vertex. Such a disk $U$ is an example of what Lackenby calls a parabolic compression disk. In other words, diagrammatically compressible surfaces are also parabolically compressible (see [22, Page 209] for a definition). For annuli, the converse also holds: by [13, Proposition 4.21], a parabolically compressible annulus $A \subset M_{A}$ must be diagrammatically compressible. Because we will be working with annuli below, we will not need the notion of parabolic compressibility in this paper.

Lemma 3.3. Let $Q$ be a square in the upper polyhedron, glued to normal squares in lower polyhedra at each of its white sides. If the white sides of $Q$ come from different polyhedral regions, then they each cut off a single ideal vertex in that white face. Hence $Q$ diagrammatically compresses in both of its white faces in the upper polyhedron.

Proof. This is a restatement of [13, Proposition 4.13].

In this paper, normal squares appear in decompositions of essential annuli. A square $Q$ in the upper polyhedron will be glued to normal squares in the lower polyhedra at each of its white sides, and so Lemma 3.3 will be useful.

The next two sections include two cases: first, that the squares making up the annulus are diagrammatically incompressible, and second, that they are diagrammatically compressible. Lemma 3.3 puts restrictions on the diagrammatically incompressible case, and so we investigate such annuli first. 

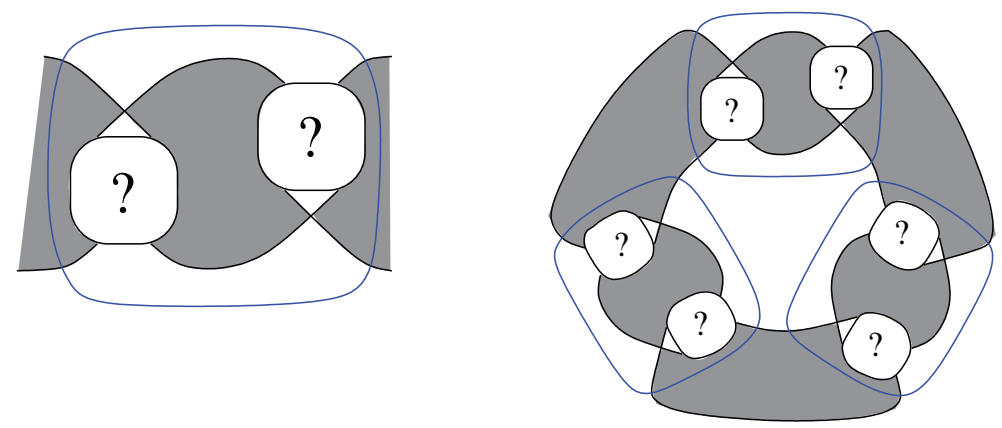

Figure 9: A fused unit and an example of a cycle of three fused units.

\section{Diagrammatically incompressible annuli}

In this section, we determine the form of any essential annulus that intersects a polyhedral region in a diagrammatically incompressible way. Then, in Lemma 4.4, we determine how diagrammatically incompressible annuli can fit into essential tori in the link complement. We emphasize that all of the results of this section work for general $A$-adequate diagrams, without any extra hypotheses.

Following Lackenby [22], we define a fused unit to be a portion of a checkerboard colored graph with the following property. Its boundary is an essential square, with two opposite sides in black regions and the other two sides each intersecting a white region adjacent to a crossing, as on the left of Figure 9. In the figure, the question marks can represent any checkerboard graph corresponding to an alternating tangle.

Lemma 4.1. Let $D(K)$ be an $A$-adequate diagram, and let $(E, \partial E) \subset$ $\left(M_{A}, \widetilde{S_{A}}\right)$ be a diagrammatically incompressible annulus. Then $E$ lies in a single polyhedral region, and the lower polyhedron in that region is a cycle of $n \geq 2$ fused units. Moreover, portions of $E$ that lie in the lower polyhedron are squares encircling a fused unit, as the blue curves in Figure 9.

Proof. Put $E$ into normal form with respect to the polyhedral decomposition of $M_{A}$. This will cut $E$ into a sequence of squares, alternating in upper and lower polyhedra.

Consider a square $S \subset E$ in the upper polyhedron. If the two white sides of $S$ lie in different polyhedral regions, Lemma 3.3 implies that it cuts off an ideal vertex in some white face, contradicting the hypothesis 
of diagrammatic incompressibility. Hence all squares of $E$ have their white sides in a single polyhedral region.

For a square $S \subset E$ in the upper polyhedron, Lemma 2.12 lets us apply the clockwise map and obtain a square $\phi(S)$ in the lower polyhedron. Consider these squares, as well as the squares of $E$ originally in the lower polyhedron. Label the squares in the lower polyhedron $S_{1}, S_{2}, \ldots, S_{2 n}$, where $S_{i}$ with even $i$ are the clockwise images of squares from the upper polyhedron.

By [13, Lemma 4.10], if any pair of adjacent squares $S_{k}$ and $S_{k+1}$ does not have intersecting white sides, then those squares must cut off single vertices in each of their white sides, implying both are diagrammatically compressible to essential product disks, which again contradicts the hypotheses.

So suppose that each $S_{k}$ and $S_{k+1}$ intersect in one, hence by [13, Lemma 4.10], both white sides. By Lemma 2.12, the white sides of odd-numbered squares differ from those of even-numbered squares by a clockwise rotation. This allows us to sketch the form of the diagram, essentially following Lackenby's proof of [22, Theorem 14].

First, suppose there are just two squares $S_{1}$ and $S_{2}$. Then they intersect in exactly two white faces of the same lower polyhedron. In each white face, $S_{2}$ differs from $S_{1}$ by a single clockwise rotation, as shown in Figure 10, left. Moreover, the white sides of the squares glue up with orientations shown in that figure. But now, note that these two squares glue to form a Möbius band, not an annulus, which is a contradiction.

So there must be at least four squares. Then $S_{1}$ differs from $S_{2}$ in one of its white faces by a single clockwise rotation, and $S_{1}$ differs from $S_{2 n}$ in the other white face of $S_{1}$ by a single clockwise rotation. The fact that $S_{2}$ and $S_{2 n}$ are disjoint [13, Lemma $\left.4.8(3)\right]$ implies that they must lie in the lower polyhedron as shown in Figure 10, right. Note this implies that $S_{1}$ bounds a fused unit.
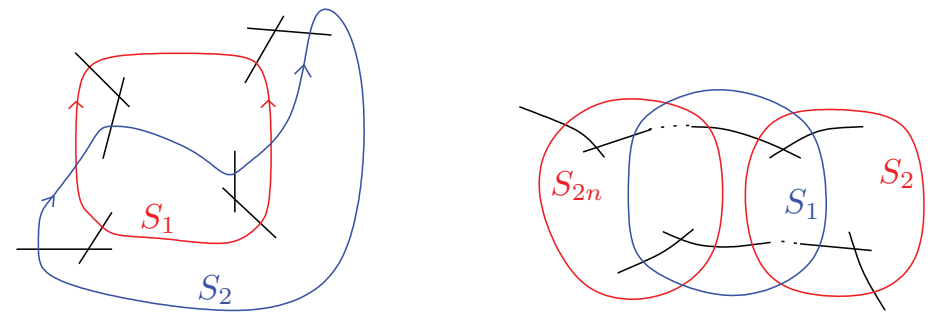

Figure 10: Left: If annulus is formed of only two squares. Right: Squares $S_{1}$, $S_{2}$, and $S_{2 n}$ must be as shown. 
Applying the same argument to $S_{3}, S_{4}$, and $S_{2}$, and continuing through all squares with odd indices, we find each odd square bounds a fused unit, and these are arranged in a cycle as claimed in the lemma.

Lemma 4.2. Let $D(K)$ be an $A$-adequate diagram. Suppose $(E, \partial E) \subset$ $\left(M_{A}, \widetilde{S_{A}}\right)$ is an embedded essential annulus such that $E$ is diagrammatically incompressible. Then there is a solid torus $V \subset M_{A}$ whose boundary consists of $E$ and an annulus $F \subset \widetilde{S_{A}}$.

Further, each of the annuli $E, F \subset \partial V$ winds once around the meridian of $V$ and $n$ times around the longitude of $V$, for the same integer $n \geq 2$ as in Lemma 4.1. In other words, the two curves of $E \cap F$ have slope $1 / n$ on the boundary of $V$.

Proof. By Lemma 3.1, white faces of the polyhedral decomposition of $M_{A}$ cut $E$ into squares, alternating between lying in the upper and lower polyhedra. Denote the squares by $S_{1}, S_{2}, \ldots, S_{2 n}$, where $S_{i}$ for even $i$ lies in the upper polyhedron. (Note that in the previous proof, $S_{i}$ for even $i$ indicated the images of these squares in the lower polyhedron.)

By Lemma 4.1, the lower polyhedron $P$ associated to $E$ is a cycle of at least two fused units, as in Figure 9. Moreover, the squares $S_{i}$ for $i$ odd encircle a fused unit.

Now recall from Section 2.2 that a lower polyhedron corresponds to a polyhedral region, i.e. a nontrivial region of the complement of the state circles and a maximal collection of non-prime arcs. A white face in a lower polyhedron $P$ is glued via homeomorphism to exactly one white face in the upper polyhedron. However, shaded faces are not glued. In a region of the upper polyhedron corresponding to a shaded face of $P$, there may be additional segments and state circles in the graph $H_{A}$. However, we may use information on white faces, as well as positions of state circles appearing in the given lower polyhedron, to sketch portions of the graph $H_{A}$. Figure 11 shows the most general possible graph.

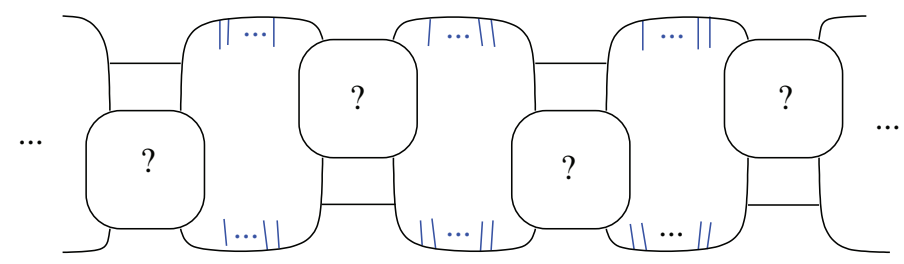

Figure 11: The graph $H_{A}$ must have the form shown when one of the lower polyhedra is a cycle of fused units. 


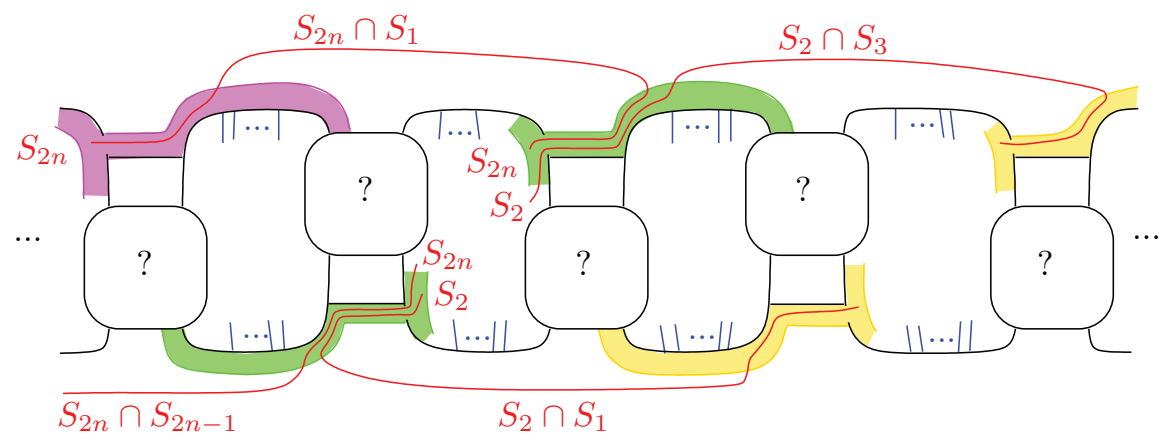

Figure 12: Upper polyhedron with squares $S_{2 k}$ is as above.

Note in particular that the white faces above and below the diagram in Figure 11 are mapped to the white faces inside and outside the cycle of fused units. Hence they only meet segments of $H_{A}$ that correspond to ideal vertices of the lower polyhedron. Thus the only possible segments in these white faces are either between state circles as shown, or inside the blocks labeled with question marks. However, a priori, there may be segments on the other sides of the state circles meeting these two white faces. Such segments are illustrated in blue in Figure 11.

The combinatorics of the upper polyhedron can be read off of the graph $H_{A}$, as described in [13, Chapter 2] or [14]. What is relevant to this discussion is that shaded faces run along segments in so-called tentacles. A tentacle is a portion of shaded face that begins on one side of a state circle (the "head"), runs along the right side of a segment when the head is oriented to be up, and then runs to the right along the adjacent state circle until it terminates at a segment. Examples of tentacles in different colors are shown in Figure 12.

The boundaries of these tentacles make up the edges of the white faces in our fused units. Hence, we use what we know of the positions of white sides of $S_{i}$ for $i$ odd to sketch white sides of $S_{j}$ for $j$ even into the upper polyhedron. In particular, white sides in the lower polyhedron are glued to those in the upper, with $S_{2}$ glued to $S_{1}$ on one side, and $S_{3}$ on the other side. Therefore, these edges are as shown in Figure 12. A pair of endpoints of white edges connect to an edge in a shaded face. Thus we may color the shaded faces at the ends of white edges in the same color. This is also shown in Figure 12.

Consider the shaded face shown in the center of Figure 12, which is shaded green. Note that $S_{2 n}$ and $S_{2}$ must both run through this green face, 
and both meet the same pair of tentacles on either side. Because shaded faces are simply connected [13, Theorem 3.12], these arcs on $S_{2}$ and $S_{2 n}$ must run parallel to each other, bounding a strip of green shaded face between them. The same argument implies $S_{2 k}$ and $S_{2(k+1)}$ bound a strip of shaded face between them, for all $k$.

Now, notice that squares $S_{i}$ with even index cut out a prism from the upper polyhedron. This prism has $n$ sides coming from the $S_{2 k}$, an additional $n$ sides coming from these strips of shaded face, and a top and bottom white face, each a polygon with $2 n$ sides.

From Lemma 4.1 and Figure 9, we see that the $S_{i}$ with odd index also cut out a prism from the lower polyhedron, with $n$ sides coming from squares $S_{2 i+1}$, and $n$ sides coming from strips of shaded face, and a top and bottom white face, each a polygon with $2 n$ sides.

These two prisms glue along the white faces to give a solid torus $V$, with boundary consisting of the annulus $E$, as well as an annulus $F \subset \widetilde{S_{A}}$ obtained by gluing the strips coming from shaded faces end to end. The white faces of the prisms form meridian disks of the solid torus $V$.

Observe from Figure 12 that the annulus $E$ intersects each meridian disk $n$ times. Observe as well that as we travel around $E$, the square $S_{2 n}$ in the upper polyhedron is glued to $S_{1}$ in the lower, then $S_{2}$ in the upper polyhedron, and so on — with $S_{2 n}$ differing from $S_{2}$ by a single $1 / n$ clockwise twist of the white face. Therefore, the annulus $E$ composed of these squares will intersect the longitude of $V$ once. Since the core curve of $E$ intersects the meridian $n$ times and the longitude once, with clockwise twisting, its slope on $\partial V$ is $1 / n$. The core curve of $F$ is parallel to that of $E$, and has the same slope.

Now recall that we are interested in an essential torus $T$, which is cut into annuli in $M_{A}$ by Lemma 3.1 .

Definition 4.3. Let $T \subset S^{3} \backslash K$ be an essential torus, decomposed into annuli as in Lemma 3.1. We say that an annulus $E \subset M_{A}$ is adjacent to $E^{\prime} \subset M_{A}$ if there is a single annulus in $N\left(S_{A}\right)$ between them.

Lemma 4.4. Let $D(K)$ be an $A$-adequate diagram. Let $T \subset S^{3} \backslash K$ be an essential torus in $S^{3} \backslash K$, and let $E, E^{\prime} \subset T \cap M_{A}$ be adjacent annuli. Then at least one of $E, E^{\prime}$ must be diagrammatically compressible. In particular, if $E$ is adjacent to itself, then it must be diagrammatically compressible.

Proof. Suppose $E$ is diagrammatically incompressible. We will examine more closely the solid torus $V$ and the annulus $F$ of Lemma 4.2 . Since $T$ is a 
torus in $S^{3}$, it is separating. Let $X$ be the component of $\left(S^{3} \backslash K\right) \backslash \backslash T$ that contains $V$, and let $X \backslash \backslash S_{A}$ denote the remnants of $X$ in $S^{3} \backslash \backslash S_{A}$. The manifold $X \backslash \backslash S_{A}$ contains $V$ as one of its components. The annulus $F \subset \widetilde{S_{A} \backslash \backslash T}$ projects to some component $R$ of $S_{A} \backslash \backslash T$.

Case 1. Suppose $R$ is orientable. Then it is an annulus. When we glue $\widetilde{S_{A}}$ to itself, to undo the cutting along $S_{A}$ and recover $S^{3} \backslash K$, the annulus $F \subset \widetilde{S_{A}} \cap \partial V$ must be glued to another annulus $F^{\prime}$, which is the boundary of some other component of $X \backslash \backslash S_{A}$. Assuming that the adjacent annulus $E^{\prime} \subset T \cap M_{A}$ is also diagrammatically incompressible, Lemma 4.2 implies there must be a solid torus $V^{\prime} \subset X \backslash \backslash S_{A}$, with boundary $\partial V^{\prime}=E^{\prime} \cup F^{\prime}$, such that the core curve of $E^{\prime}$ has slope $1 / n$. By Lemma $4.1, n$ is at least 2. But then $\partial E$ and $\partial E^{\prime}$ are both glued onto $\partial R$, hence $E$ and $E^{\prime}$ form all of $T \cap M_{A}$. Hence $X$ consists of exactly two solid tori, glued along annuli of slopes $1 / n_{i}$ on their respective boundaries.

Case 2. Suppose $R$ is non-orientable. Then, since $F \subset \partial N(R) \subset \widetilde{S_{A}}$ is a double cover of $R$, it follows that $R$ must be a Möbius band. To form $S^{3} \backslash K$ from $S^{3} \backslash \backslash S_{A}$, we glue appropriate boundary components of $N\left(S_{A}\right)$. The annulus $F$, as the boundary of a regular neighborhood of a Möbius band, must be glued to itself, with the regular neighborhood $N(R)$ collapsing onto $R$. But then under this gluing, the boundary components of the annulus $E$ are glued only to boundary components of the annulus $E$. This means $S_{A}$ cuts $T$ into only the annulus $E$, and $V$ is the only component of $X \backslash \backslash S_{A}$. Therefore, $X$ must be the result of gluing $V$ to itself along an annulus with slope $1 / n$ on its boundary.

Here is another way to think of this gluing. The regular neighborhood $N(R) \subset N\left(S_{A}\right)$ is a solid torus. Since $F$ double-covers the Möbius band $R$ and runs around this solid torus twice, the slope of $F$ on $\partial N(R)$ is $1 / 2$. We conclude that $X$ is obtained by gluing together two solid tori along annuli whose slopes are $1 / n$ and $1 / 2$.

In both cases, $X$ consists of two solid tori $V_{1}$ and $V_{2}$, glued along annuli of slopes $1 / n_{i}$, where each $n_{i}$ is at least 2 . This means that $X$ is a Seifert fibered space with base space a disk and two singular fibers. (See Hatcher [19] for background.)

Next, we will calculate the Seifert invariants of $X$. For simple, oriented curves $x, y$ on $T_{i}=\partial V_{i}$, let $\langle x, y\rangle$ denote their algebraic intersection number. Also abusing notation, we will use the same symbol to denote a curve on $T_{i}$ and its homology class in $H_{1}\left(T_{i}\right)$. Let $Q_{i}, H_{i}$ denote a pair of a cross-section curve and fiber of the fibration on $T_{i}$, oriented so that $\left\langle Q_{i}, H_{i}\right\rangle=1$. Let $\mu_{i}, \lambda_{i}$ be a meridian and longitude of $T_{i}$, chosen so that $\mu_{i}$ bounds a disk 
in $V_{i}$ and $\left\langle\mu_{i}, \lambda_{i}\right\rangle=1$. Furthermore, we can normalize $\lambda_{i}$ so that the gluing annulus, which is foliated by fibers parallel to $H_{i}$, satisfies $H_{i}=\mu_{i}+n_{i} \lambda_{i}$. Since $Q_{i}=a_{i} \mu_{i}+b_{i} \lambda_{i}$ for some coefficients $a_{i}, b_{i}$, it follows that

$$
\left\langle Q_{i}, H_{i}\right\rangle=n_{i} a_{i}-b_{i}=1
$$

Solving for $\mu_{i}$, we have $\mu_{i}=n_{i} Q_{i}-b_{i} H_{i}$. Thus the slope of each singular fiber of $X$ is $-b_{i} / n_{i}$, making the Euler number of $X$ equal to

$$
e=-\frac{b_{1}}{n_{1}}-\frac{b_{2}}{n_{2}}=\frac{-n_{2} b_{1}-b_{2} n_{1}}{n_{1} n_{2}} .
$$

Since $T=\partial X$ is incompressible, $X$ cannot be a solid torus. Thus $X$ admits a unique Seifert fibration [19, Theorem 2.3], which means that its Seifert invariants are uniquely determined modulo 1 [19, Proposition 2.1]. Since $X$ embeds in $S^{3}$ and $\partial X$ is a single torus, $X$ must be the complement of a torus knot, say $H$. On $\partial X, H$ may be identified with a regular fiber of the fibration of $X$. Let $\mu, \lambda$ denote the meridian and canonical longitude of $\partial X$, again with the convention that $\langle\mu, \lambda\rangle=1$. Since the geometric intersection of the meridian and $H$ is 1 , if $Q$ is a cross section curve on $\partial X$, then

$$
\mu=Q+x H
$$

so that $\langle\mu, H\rangle=\langle Q, H\rangle=1$. A minimum genus Seifert surface for $\lambda$ will be horizontal with respect to the Seifert fibration of $X$. By the Claim in the proof of [19, Proposition 2.2], the boundary slope of this surface with respect to the $(Q, H)$ framing is equal to the Euler number $e$. Therefore, by Equation (2),

$$
\lambda=n_{1} n_{2} Q+\left(-n_{2} b_{1}-b_{2} n_{1}\right) H .
$$

Since $\langle\mu, \lambda\rangle=1$, Equations (3) and (4) imply

$$
-x n_{1} n_{2}-n_{2} b_{1}-b_{2} n_{1}=1
$$

By (1), we obtain $n_{1} n_{2} a_{1}+n_{2} n_{1} a_{2}-\left(n_{1} b_{2}+b_{1} n_{2}\right)=\left(n_{1}+n_{2}\right)$, which combined with (5) gives

$$
n_{1} n_{2}\left(a_{1}+a_{2}+x\right)+1=\left(n_{1}+n_{2}\right) \quad \Rightarrow \quad a_{1}+a_{2}+x=\frac{1}{n_{1}}+\frac{1}{n_{2}}-\frac{1}{n_{1} n_{2}},
$$

which is impossible since $a_{i}, x$ are integers and $n_{i} \geq 2$. 
Remark 4.5. Lemma 4.4 can also be proved using diagrammatic techniques. The idea is as follows. From Figures 9 and 12, one can determine how the annulus $F \subset \widetilde{S_{A}}$ lies in the diagram. If two annuli $F$ and $F^{\prime}$ as above are mapped to a single annulus in $S_{A}$ (Case 1 of the above proof), then their cores must map to the same curve on $S_{A}$. A careful analysis of annuli $F$ and $F^{\prime}$, each lying in the diagram as specified by Figures 9 and 12 , yields a contradiction. Similarly, if $F$ is glued to itself when $\widetilde{S_{A}}$ is mapped to $S_{A}$ (Case 2 of the above proof), then the core of $F$ must wrap around the same curve on $S_{A}$ twice. Again a diagrammatic analysis will reveal that this is impossible.

\section{Diagrammatically compressible annuli}

Lemma 4.4 implies that the intersection of an essential torus with the corresponding polyhedral decomposition must contain components that intersect the decomposition in a diagrammatically compressible way. In this section, we determine information on such annuli under the hypothesis of Theorem 1.1 .

Suppose an annulus decomposes into a square that is diagrammatically compressible in one white face, i.e. it cuts off a single ideal vertex there. Then it must be diagrammatically compressible in every white face, either by Lemma 3.3, or by an application of the clockwise map, Lemma 2.12, and [13, Lemma 4.10]. Diagrammatically compressible squares are closely related to essential product disks.

Definition 5.1. An essential product disk, or EPD, is a properly embedded essential disk in $M_{A}$ whose boundary meets the parabolic locus of $M_{A}$ twice.

When an essential product disk $R$ lies in a single polyhedron in the polyhedral decomposition of $M_{A}$, we may think of it as a quadrilateral with two sides on shaded faces, coming from $\widetilde{S_{A}}$, and two sides running over ideal vertices of the polyhedron, which correspond to the parabolic locus. We may pull $R$ off the ideal vertices into adjacent white faces, obtaining a normal square $Q$, with two sides on shaded faces and two sides on white faces. The two sides of $Q$ on white faces each cut off a single ideal vertex; that is, the square is diagrammatically compressible in both of its white faces. Conversely, if $Q$ is a normal square, each of whose white sides cuts off a single ideal vertex of the ambient white face, then pulling $Q$ onto those ideal vertices (that is, performing a parabolic compression) produces either an EPD or a square encircling a single ideal vertex. 

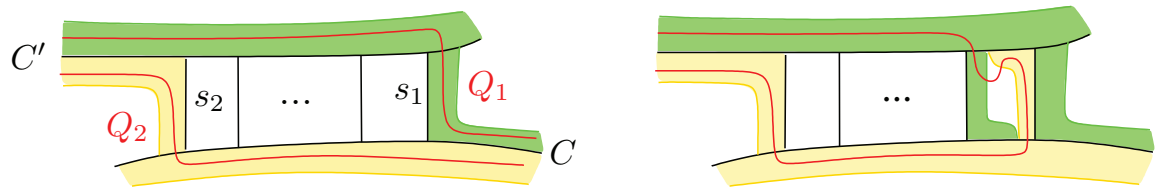

Figure 13: Possible forms of squares parabolically compressing to an EPD. The square on the left is called a square in a twist region (Definition 5.5). The right-most white face satisfies Convention 5.3.

Remark 5.2. Throughout this section, we will assume that the sides of normal squares in shaded faces run monotonically through tentacles, without unnecessary backtracking. This assumption can be easily satisfied by normal isotopy. The precise terminology from [13] is that the sides of squares are simple with respect to the shaded faces, as in [13, Definition 3.2].

Convention 5.3. Let $Q$ be a normal square, and $W$ a white face in which $Q$ cuts off a single ideal vertex. We may color the shaded faces met by $Q$ orange and green, so that the single vertex of $W$ cut off by $Q$ is a triangle whose three edges, in counter-clockwise order, are orange-green-white. For instance, the right-most white face in Figure 13 satisfies this convention.

The next lemma places strict restrictions on the form of a square coming from an EPD in the upper polyhedron. The proof relies on the hypothesis that 2-edge loops in $\mathbb{G}_{A}$ belong to twist regions (Definition 2.5), as well as the classification of EPDs into seven combinatorial types [13, Theorem 6.4]. This proof is likely the most technical argument of this paper, and can be omitted without missing the thread of the argument.

Lemma 5.4. Suppose a prime, A-adequate diagram is such that $\mathbb{G}_{A}$ satisfies the 2-edge loop condition. Suppose that a normal square $Q$ parabolically compresses to an EPD in the upper polyhedron. Then, possibly after sliding one white side of $Q$ past an ideal vertex, we obtain a normal square $P$ with one of the two forms shown in Figure 13. In particular, the boundary of $P$ runs over distinct segments of the same twist region, with two sides on shaded faces adjacent to the same state circle on a side of that twist region.

Proof. Given a diagrammatically compressible square $Q$, select one of the sides that cuts off a single ideal vertex in a white face $W$. Color the two adjacent shaded faces according to Convention 5.3.

With this labeling of shaded faces, consider the side of $Q$ in its other white face, $W^{\prime}$. This side also cuts off a single ideal vertex, but that ideal 
vertex may be a triangle with opposite orientation compared to that of Convention 5.3. If this occurs, replace $Q$ with a new square $P$ by sliding the white side $Q \cap W^{\prime}$ across the adjacent ideal vertex, so that it lies in a new white face $W^{\prime \prime}$ and cuts off a triangle oriented in the opposite direction. This new square $P$ must satisfy Convention 5.3 in both of its white faces.

We now apply [13, Theorem 6.4], which applies to normal squares such as $P$ that satisfy Convention 5.3 in both white faces (equivalently, satisfy [13, Lemma 6.1]). This theorem implies that the normal square $P$ is of one of seven types, shown in [13, Figure 6.1]. Assuming that 2-edge loops of $\mathbb{G}_{A}$ belong to twist regions, we may rule out all these types except the first two, labeled $\mathcal{A}$ and $\mathcal{B}$ in $[13$, Figure 6.1]. These are exactly the two types shown in Figure 13. We now describe how to rule out the remaining types.

Type $\mathcal{C}$. Suppose the normal square is of type $\mathcal{C}$, reproduced in Figure $\overline{14}$, left. The 2-edge loop shown belongs to a twist region by hypothesis, and so a twist region must lie either on the inside of the segments shown or the outside. Note there is a segment meeting the lower state circle on the opposite side of the 2-edge loop on the inside. This cannot happen if the twist region lies on the inside. Hence the twist region must lie on the outside of the two segments shown, as in Figure 14, middle. Then the orange face must run all the way across the outside of the twist region, meeting no tentacles or non-prime arcs, as shown in that figure. Draw an arc through the orange face all the way across the twist region. On the far side, connect the arc across the state circle to the portion of the square $P$ in the green face. Now continue to follow $P$ to the right. It crosses the state circle once more, before joining the orange face where we began, as in Figure 14, right. Replace segments of $H_{A}$ by crossings. We obtain a simple closed curve in the diagram of the link that meets the link exactly twice, with crossings on either side. This contradicts the hypothesis that the diagram is prime.

In the interest of space, we do not reproduce [13, Figure 6.1] for the analysis of the rest of the cases. However, all the cases are handled by a variation of the above argument.

Type $\mathcal{E}$. This can also be ruled out by an appeal to the primeness of the diagram. Because the 2-edge loop in type $\mathcal{E}$ comes from a twist region, the orange face shown, adjacent to the state circle at the bottom, must continue across the bottom of that state circle, meeting no segments or non-prime arcs, until it lies directly opposite the green face running next to the second sement of the 2-edge loop. Note that the orange face is also directly opposite the green face on the left side of the green non-prime arc. Now we may draw an arc through the orange face, from the point where it is opposite the 

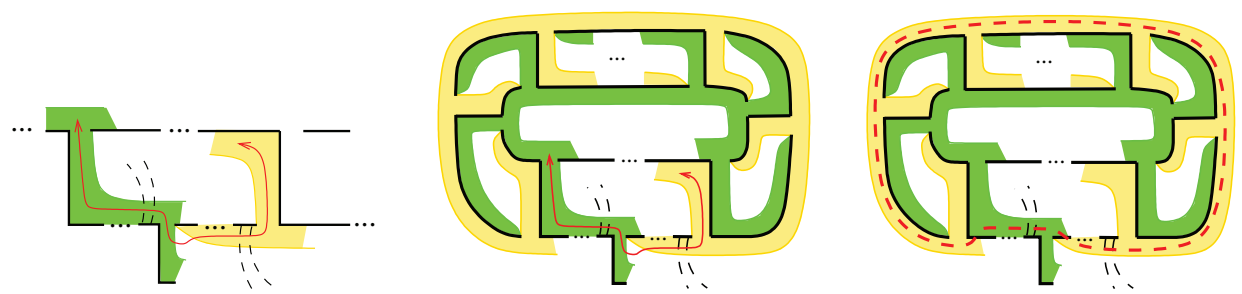

Figure 14: Left: A normal square of type $\mathcal{C}$ runs over a portion of the upper polyhedron of the form shown. Middle: The precise form imposed by 2 -edge loop condition. Right: Dotted line gives a contradiction to the primeness of the diagram.

non-prime arc to the point where it is opposite the segment of the 2-edge loop. Connect the arc across the state circle to the portion of $P$ lying in the green face. This gives a simple closed curve meeting $H_{A}$ exactly twice on state circles, which in turn gives a loop in the diagram meeting the diagram exactly twice, again contradicting the hypothesis that the diagram is prime.

Type $\mathcal{G}$. The segments forming the 2-edge loop shown in that figure must belong to a twist region by hypothesis, meaning there can be only segments and state circles of the short resolution of a twist region between those two segments on one side. But on one side of the two segments there is a segment on the opposite side of the state circle, and on the other side there is a non-prime arc. Hence type $\mathcal{G}$ cannot occur.

Types $\mathcal{D}$ and $\mathcal{F}$. Because the 2-edge loop shown belongs to a twist region, one green tentacle must terminate in a bigon, with no additional green tentacles or non-prime arcs connected to it. For type $\mathcal{D}$, this green tentacle lies to the right in [13, Figure 6.1]. For type $\mathcal{F}$, it lies ot the left. In either case, since $P$ runs through that tentacle, it must meet its second ideal vertex at the end of that green tentacle shown. In other words, at the end of that green tentacle it must cut off a single vertex in a white face, which defines a triangle. However, note that the triangle will have the opposite orientation from Convention 5.3, contradicting our assumption.

It follows that our normal square $P$ is of type $\mathcal{A}$ or $\mathcal{B}$. To obtain Figure 13 , we make the following observations. First, because the 2-edge loop shown in either figure belongs to a twist region, the two segments shown must bound a string of bigons, i.e. segments and state circles from the short resolution of a twist region. In particular, there can be no segments on the opposite side of the state circles shown between these two segments. This 
implies that some face runs straight across the top of the twist region, meeting no additional segments or state circles, and the orange face runs straight across the bottom of the twist region, meeting no additional segments or state circles.

For type $\mathcal{A}$, the green face must run across the top of the twist region. If the boundary of $P$ runs into the green face at the top, then it must run straight across the top, and straight across the bottom through the orange face, and hence $P$ is as shown on the left of Figure 13. It may be the case for type $\mathcal{A}$ that the boundary of $P$ runs down a green tentacle, without running across the top. Then in this case, a portion of $P$ in the orange face runs between these segments, so the two segments form a bigon, and the EPD must meet the ideal vertices of the bigon. Given our choice of orientation, the normal square $P$ will have the form of Figure 13, right.

For type $\mathcal{B}$, we need to show that the green face runs across the top of the twist region. This follows because the portion of $P$ adjacent to the orange segment on the right is running downstream in a tentacle which forms a bigon of the twist region. No other tentacles or non-prime arcs can meet this bigon, or the 2-edge loop would not belong to a twist region. Hence $P$ meets the white face at the end of this tentacle, and joins the green on the opposite side. It follows that the green face must run across the twist region, with $P$ running across as well to meet in this vertex. Thus the normal square $P$ is as shown on the right of Figure 13 .

The square on the left of Figure 13 has a particularly relevant form.

Definition 5.5. A diagrammatically compressible square $Q$ with sides $Q_{1}$, $Q_{2}$ in shaded faces is defined to be a square in a twist region $R$ if it is of the form shown on the left of Figure 13. More precisely, there are state circles $C$ and $C^{\prime}$ and segments $s_{1}, s_{2}$ of $H_{A}$ between $C$ and $C^{\prime}$, determining $R$, such that:

(i) $Q_{1}$ and $Q_{2}$ run on adjacent shaded faces near $C^{\prime}$;

(ii) $Q_{1}$ and $Q_{2}$ run on adjacent shaded faces near $C$;

(iii) one side of $Q$, say $Q_{1}$, runs on the top of the twist region along $C^{\prime}$ and then along $s_{1}$, while $Q_{2}$ runs along $s_{2}$ and then on the bottom of $R$ along $C$.

Lemma 5.6. Suppose a prime, A-adequate diagram is such that $\mathbb{G}_{A}$ satisfies the 2-edge loop condition. Suppose $T \subset S^{3} \backslash K$ is an essential torus, $E \subset T \cap M_{A}$ is a diagrammatically compressible annulus, and $Q \subset E$ is a 
normal square that parabolically compresses to an EPD in the upper polyhedron. Then we may perturb $E$ by a normal isotopy that extends to $T$, so that $Q$ becomes a square in a twist region.

Proof. Suppose $Q$ parabolically compresses to an EPD. Then, by Lemma 5.4, there is a related normal square $P$ that has one of the two forms of Figure 13. For each of the forms, $P$ may either agree with $Q$ or be obtained from $Q$ by moving one white side across an ideal vertex of the upper polyhedron.

Suppose $P$ is as on the left of Figure 13. If $P$ and $Q$ agree, then we are finished. Otherwise, $P$ differs from $Q$ in that one of its white sides is on the opposite side of an ideal vertex from $Q$. We may assume that this white side is located to the right of the figure shown. If the ideal vertex in question does not contain the right-most segment of the twist region of Figure 13, left, then adjusting $P$ to form $Q$ has no effect on the region shown, and the diagram is as claimed. If the ideal vertex does contain the right-most segment, then $Q$ must have a white side on the opposite side of the corresponding ideal vertex, which lies in the right-most bigon of the twist region shown, and $Q$ now has the form of a square on the right of Figure 13.

Now suppose that $Q$ is as shown on the right of Figure 13, and either $P$ agrees with $Q$, or $P$ is as on the left of Figure 13, as in the previous paragraph. Then the white side of the square on the right of the figure lies in a bigon white face. This white face is glued to a bigon in a lower polyhedron, and the annulus runs through the bigon. Isotope the annulus through the bigon, isotoping $Q$ to have a white side cutting off a vertex on the opposite side of the bigon. This pulls the shaded sides of $Q$ along as well, pulling the side of $Q$ in the right-most orange tentacle to only meet the head of that tentacle, and pulling the side in the green to run downstream adjacent to a green tentacle. The isotopy can be performed in a neighborhood of the white face in $M_{A}$, affecting only the square $Q$ and the square in the lower polyhedron glued to $Q$ at this face, but only in a neighborhood of this single white face. Moreover, the isotopy may be extended into a small neighborhood of $\widetilde{S_{A}}$, to extend to all of $T$. After this isotopy, $Q$ has the form claimed in the lemma.

The only remaining case is that the square $P$ has the form on the right of Figure 13, but it differs from the original square $Q$ in that we slid a white side of $Q$ through an ideal vertex. Because we were able to choose the orientation on one vertex, we may assume that the ideal vertex in question is at the right of that figure. Notice that the side of $P$ that must be adjusted lies in a bigon in a twist region, with state circle $C^{\prime}$ on top, and $C$ on bottom of the twist region. The ideal vertex cut off by $P$ is a portion of the graph 
$H_{A}$ consisting of a small arc on $C^{\prime}$, a segment $s$ connecting $C^{\prime}$ and $C$, a portion of $C$, and possibly more segments. Hence when we slide over this ideal vertex, the portion of $P$ running through the orange tentacle adjacent $s$ slides out of this tentacle, and a portion of the square in the green face will be pulled through the green tentacle adjacent to $s$. When finished, the result will be as claimed in the statement of the lemma.

The next lemma will allow us to show that, assuming the 2-edge loop condition, no diagrammatically incompressible annuli in $M_{A}$ come from essential tori. This is done in Lemmas 5.8 and 5.9.

Lemma 5.7. Let $D(K)$ be an $A$-adequate diagram, with all-A state surface $S_{A}$, and let $T$ be an incompressible torus. Suppose that a normal square $Q \subset T \cap M_{A}$ lies in the upper polyhedron, with sides $Q_{1}$ and $Q_{2}$ in shaded faces, which satisfies the following property. There exists a point $p$ on a state circle and arcs $q_{1} \subset Q_{1}$ and $q_{2} \subset Q_{2}$ that can be isotoped to lie on either side of an $\epsilon$ neighborhood of $p$, while maintaining the condition that $Q_{1}$ and $Q_{2}$ are simple with respect to their shaded faces (Remark 5.2).

Then $T \backslash \widetilde{S_{A}}$ consists of exactly two annuli, one in $M_{A}$ and one in the I-bundle $N\left(S_{A}\right)$. Moreover, subarcs of $q_{1}$ and $q_{2}$ are glued together in $T$ when we glue opposite sides of $N\left(S_{A}\right)$, to recover $S^{3} \backslash K$.

Proof. The point $p$ on a state circle corresponds to a point, which we will also call $p$, on the knot $K$. Let $N_{p}$ be a tubular neighborhood of $p$ in $S^{3}$. We may take this neighborhood small enough that $N_{p} \cap M_{A}$ lies entirely in the upper polyhedron, and $N_{p} \cap N\left(S_{A}\right)$ is a trivial $I$-bundle, of the form $D \times[-1,1]$ for some disk $D$ in $S_{A}$. Moreover, we may isotope $q_{1}$ and $q_{2}$ (and all of $T$ ), if necessary, so that $q_{1}$ and $q_{2}$ each run through $N_{p}$. Since $Q$ has opposite sides containing $q_{1}$ and $q_{2}$, and since $Q$ lies in a ball (the upper polyhedron), we may isotope $Q$ relative to its boundary so that a sub-rectangle $Q^{\prime}$ of $Q$ has opposite sides on $q_{1}$ and $q_{2}$, and lies completely in $N_{p}$.

Now consider $Q^{\prime}$. This is a rectangle in the tube $N_{p}$ surrounding the point $p$ on the knot $K$. It has one boundary component, say $q_{1}$, on $D \times\{-1\}$, and one boundary component, $q_{2}$, on $D \times\{1\}$. Notice it almost encircles $K$ to form an annulus whose core is a single meridian, except that it is cut by $N\left(S_{A}\right)$. Inside $N\left(S_{A}\right)$, both $q_{1}$ and $q_{2}$ are glued to annuli which are isotopic to vertical annuli in the $I$-bundle. The proof will be complete when we show that $q_{1}$ and $q_{2}$ are glued to the same vertical annulus in the $I$-bundle. 
So suppose not. Suppose $q_{1}$ is glued to the annulus $E_{1}$ and $q_{2}$ is glued to the annulus $E_{2}$. Since $E_{1}$ and $E_{2}$ are both subsets of $T$, they are disjoint. They are also compact, connected, and they lie inside the compact set $N\left(S_{A}\right) \backslash N_{\delta}(K)$, where $N_{\delta}(K)$ is a sufficiently small tubular neighborhood of $K$. Consider the projection $\pi: N\left(S_{A}\right) \backslash N_{\delta}(K) \rightarrow S_{A}$. This is a continuous map on a compact set, hence $\pi\left(E_{1}\right)$ and $\pi\left(E_{2}\right)$ are compact in $S_{A}$. Since $E_{i}$ is the image of a vertical annulus, it lies in a bounded neighborhood of a core curve $\tau_{i}$ on $S_{A}, i=1,2$.

Now, let $\sigma$ be a simple arc in $S_{A}$ with one endpoint on $p$, exiting $N_{p} \cap S_{A}$ by crossing through $\pi\left(E_{1}\right)$ and $\pi\left(E_{2}\right)$, and such that $\sigma$ meets each of $\tau_{1}, \tau_{2}$ transversely exactly once, and the final endpoint of $\sigma$ is disjoint from $\pi\left(E_{1}\right)$ and $\pi\left(E_{2}\right)$. Note that if we restrict the $I$-bundle $N\left(S_{A}\right)$ to $\sigma$, we obtain a trivial $I$-bundle over a line, which is a rectangle $R \cong \sigma \times[-1,1]$. Note also that $R$ intersects both $E_{1}$ and $E_{2}$ in $R \cap N_{p}$. In particular, an arc of intersection of $E_{1} \cap R$ has endpoint in $N_{p}$ at a point on $\sigma \times\{-1\}$, and an arc of intersection of $E_{2} \cap R$ has an endpoint in $N_{p}$ on $\sigma \times\{1\}$.

Consider the other endpoint of $E_{1} \cap R$. By choice of $\sigma$, this endpoint must be either on $\sigma \times\{-1\}$ or on $\sigma \times\{1\}$. If $E_{1} \cap R$ has both endpoints on $\sigma \times\{-1\}$, then there is a disk in $R$ with boundary on $E_{1} \cap R$ and on $\sigma \times\{-1\}$. We may isotope $E_{1}$ through this disk, removing the intersection of $E_{1}$ with $R$, hence pushing the arc $q_{1}$ away from $p$. Such an isotopy is impossible under the assumption that the sides of $Q$ were simple. Hence $E_{1} \cap R$ has one endpoint on $\sigma \times\{-1\}$ and one on $\sigma \times\{1\}$. Similarly, $E_{2} \cap R$ has one endpoint on $\sigma \times\{1\}$ and one on $\sigma \times\{-1\}$.

Because $E_{1}$ and $E_{2}$ are embedded, these endpoints cannot interleave. Thus either both endpoints of $E_{1} \cap R$ will lie in $N_{p} \cap R$ or both endpoints of $E_{2} \cap R$ will lie in $N_{p} \cap R$. Say both endpoints of $E_{2}$ lie in $N_{p} \cap R$.

Now, $E_{2}$ must connect to an annulus in $M_{A}$ on both of its boundary components. One boundary component connects to an annulus containing $Q$. The other cannot connect to $Q$ because its initial endpoint on $R$ lies interior to $q_{1} \cap R$. So it connects to some new rectangle $Q^{\prime \prime}$. But consider the shaded sides of $Q^{\prime \prime}$. One lies parallel to $q_{1}$, but interior to $q_{1}$ (i.e. closer to $K$ ) on $\widetilde{S_{A}}$. The other cannot also be parallel to $q_{1}$ in $N_{p}$, or we could eliminate an intersection of $T$ with $S_{A}$. But $Q^{\prime \prime}$ cannot intersect $Q$, hence $Q^{\prime \prime}$ must lie parallel to $Q^{\prime}$ in $N_{p}$, and its opposite boundary component is parallel to $q_{2}$ on $\widetilde{S_{A}}$, but closer to $K$.

Now we may repeat the entire above argument with $Q^{\prime \prime}$, to obtain a square interior to $Q^{\prime \prime}$ in $N_{p}$. Since each square we pick up at each step is interior to all previous squares, we obtain an infinite sequence of squares, each lying on $T$. This is a contradiction. 
Thus $q_{1}$ and $q_{2}$ are connected by a single annulus $E \subset N\left(S_{A}\right)$, which can be isotoped to be vertical.

Lemma 5.8. Suppose a prime, $A$-adequate is such that $\mathbb{G}_{A}$ satisfies the 2-edge loop condition. Then each diagrammatically compressible square in the upper polyhedron coming from an essential torus must bound a single ideal vertex.

Proof. Suppose we have a square $Q \subset T \cap M_{A}$ that does not encircle a single ideal vertex. Then $Q$ parabolically compresses to an EPD. By Lemma 5.6, we may isotope the torus $T$ containing $Q$ so that $Q$ is a square in a twist region. By parts (i)-(ii) of Definition 5.5, there are points $p \in C$ and $p^{\prime} \in C^{\prime}$ for which the hypotheses of Lemma 5.7 are satisfied. By the conclusion of that lemma, there is only one annulus of $T \cap M_{A}$, and the shaded sides of $Q$ that run near $p^{\prime}$ (resp. $p$ ) are glued to each other in $T$.

However, note that between these two pairs of glued arcs, the sides of the square run adjacent to distinct segments of $H_{A}: Q_{1}$ runs along $s_{1}$, while $Q_{2}$ runs along $s_{2}$. By the above paragraph, these sides of $Q$ are glued to each other when we collapse $N\left(S_{A}\right)$ to $S_{A}$ to recover the torus $T$. Hence the shaded sides of $Q$ on $\widetilde{S_{A}}$ have homotopic projection to $S_{A}$. The graph $\mathbb{G}_{A}$ is a spine for $S_{A}$, so we may homotope the arcs in $S_{A}$ to run over the same edges of $\mathbb{G}_{A}$ in the same order. But because the arcs run adjacent to distinct segments of $H_{A}$, they run over distinct edges (corresponding to these segments) in $\mathbb{G}_{A}$. This is a contradiction.

Lemma 5.9. Suppose $D(K)$ is is a prime, $A$-adequate diagram such that $\mathbb{G}_{A}$ satisfies the $2-e d g e$ loop condition. Suppose an essential torus $T$ contains a diagrammatically compressible annulus $E \subset T \cap M_{A}$. Then $E$ is the only component of $T \cap M_{A}$, and every normal square comprising $E$ encircles a single ideal vertex in its ambient polyhedron.

Proof. By Lemma 5.8, each diagrammatically compressible square in the upper polyhedron bounds a single ideal vertex. It follows from Lemma 5.7 that each such square has one side in a shaded face glued to the other side when we re-glue $N\left(S_{A}\right)$. Thus $E$ is the only annulus of $T \cap M_{A}$.

Each square $Q$ in the upper polyhedron is attached to a diagrammatically compressible square $Q^{\prime}$ in the lower polyhedron. A diagrammatically compressible square in the lower polyhedron intersects the diagram graph exactly four times, adjacent to crossings. Because the diagram is $A$-twist reduced (Lemma 2.7), the square must encircle either an ideal vertex or a string of bigons. 
Suppose the square $Q^{\prime}$ encircles a nonempty string of bigons. Because each side in a white face is glued to a square in the upper polyhedron, and each square in the upper polyhedron has shaded sides glued when we reattach sides of $N\left(S_{A}\right)$ to form $S^{3} \backslash K$, the square in the lower polyhedron must also have its sides in shaded faces glued when we form $S^{3} \backslash K$.

But now consider the way these sides run through the graph $\mathbb{G}_{A}$, which forms a spine for the surface $S_{A}$. One of the curves runs through a vertex associated with a state circle on one side of the twist region, and the other runs through a distinct vertex on the other side of the twist region. Because the sides in white faces are adjacent to distinct crossings, the curves cannot be homotopic in $S_{A}$, hence they cannot be glued in the square. This contradiction implies that the square $Q^{\prime}$ runs over a single ideal vertex.

We are now ready to show that semi-adequate links that satisfy the hypothesis of Theorem 1.1 have atoroidal complements.

Theorem 5.10. If $D(K)$ is a prime, A-adequate diagram such that $\mathbb{G}_{A}$ satisfies the 2-edge loop condition, then $S^{3} \backslash K$ contains no embedded essential tori.

Proof. Suppose $T$ is an embedded essential torus in $S^{3} \backslash K$. By Lemma 3.1, we may take $T$ to be embedded in such a way that $\widetilde{S_{A}}$ cuts it into an even number of essential annuli, half embedded in $M_{A}$ and half embedded in the $I$-bundle $N\left(S_{A}\right)$. By Lemma 4.4, $T \cap M_{A}$ must contain a diagrammatically compressible annulus $E$.

But by Lemma 5.9, if the essential torus gives rise to a diagrammatically compressible annulus $E$, then $E$ is the only component of $T \cap M_{A}$, and every normal square of $E$ encircles a single ideal vertex in its polyhedron. In that case, when we glue opposite sides of $N\left(S_{A}\right)$ to recover $S^{3} \backslash K$, the sides of $E$ will be identified to encircle knot strands, and it follows that the torus $T$ is actually boundary parallel, and not essential.

\section{Seifert fibered link complements}

Our work in the previous sections reduces the proof of the main result to the case of atoroidal link complements. As we remarked in the introduction, the diagram $D(K)$ is assumed connected, which implies that $K$ is non-split and $S^{3} \backslash K$ is irreducible. By work of Thurston [30], an irreducible, atoroidal 3 -manifold is either hyperbolic or Seifert fibered. To finish the proof of Theorem 1.1 we need to treat the case of Seifert fibered link complements. This is done in the following theorem. 
Theorem 6.1. Let $D(K)$ be a prime, connected, A-adequate diagram such that $\mathbb{G}_{A}$ satisfies the 2-edge loop condition. If $S^{3} \backslash K$ is Seifert fibered, then $D(K)$ is the standard diagram of a $(2, q)$ torus link.

Proof. Let $\mathbb{G}_{A}^{\prime}$ denote the reduced state graph of $D(K)$, obtained from $\mathbb{G}_{A}$ by removing all the duplicate edges. Recall that the guts of $S^{3} \backslash K$ relative to the surface $S_{A}$, denoted guts $\left(S^{3} \backslash K, S_{A}\right)$, is the complement of the maximal $I$-bundle in $M_{A}=S^{3} \backslash \backslash S_{A}$. In [13, Corollary 5.19] we proved that, when all 2 -edge loops in $\mathbb{G}_{A}$ belong to twist regions,

$$
-\chi\left(\operatorname{guts}\left(S^{3} \backslash K, S_{A}\right)\right)=\max \left\{-\chi\left(\mathbb{G}_{A}^{\prime}\right), 0\right\} .
$$

Furthermore, the work of Agol [4], as generalized by Kuessner [21], says that guts can be used to estimate the Gromov norm of $S^{3} \backslash K$ :

$$
\left\|S^{3} \backslash K\right\| \geq-2 \chi\left(\operatorname{guts}\left(S^{3} \backslash K, S_{A}\right)\right) \geq-2 \chi\left(\mathbb{G}_{A}^{\prime}\right) .
$$

Recall that the Gromov norm $\|M\|$ of a 3 -manifold $M$ is positive whenever the JSJ decomposition has one or more hyperbolic pieces $[5,18]$. In particular, if $S^{3} \backslash K$ is Seifert fibered, we have $\left\|S^{3} \backslash K\right\|=0$, hence $\chi\left(\mathbb{G}_{A}^{\prime}\right) \geq 0$.

Next, recall that the graph $\mathbb{G}_{A}$ can be given the structure of a ribbon graph [8], and as such it can be embedded on a standard closed orientable surface (called the Turaev surface of $D(K)$ ) so that it defines a cellulation $[8,31]$. The genus of this surface is called the Turaev genus of $D(K)$. The Turaev genus $g(D)$ satisfies

$$
\begin{aligned}
2 g(D) & =2-v\left(\mathbb{G}_{A}\right)+e\left(\mathbb{G}_{A}\right)-f\left(\mathbb{G}_{A}\right) \\
& =2-\chi\left(\mathbb{G}_{A}^{\prime}\right)+\left(e\left(\mathbb{G}_{A}\right)-e\left(\mathbb{G}_{A}^{\prime}\right)\right)-f\left(\mathbb{G}_{A}\right),
\end{aligned}
$$

where $v\left(\mathbb{G}_{A}\right), e\left(\mathbb{G}_{A}\right), f\left(\mathbb{G}_{A}\right)$ denote the number of vertices, edges and faces, respectively, of the aforementioned cellulation, and $e\left(\mathbb{G}_{A}^{\prime}\right)$ is the number of edges of $\mathbb{G}_{A}^{\prime}$.

Since 2-edge loops in $\mathbb{G}_{A}$ belong to twist regions of $D(K)$, for every edge in $\left.e\left(\mathbb{G}_{A}\right)-e\left(\mathbb{G}_{A}^{\prime}\right)\right)$ there is a bigon face in $f\left(\mathbb{G}_{A}\right)$ that cancels that edge. Furthermore, if $D(K)$ has more than one twist region - if it is not the standard diagram of a $(2, q)$ torus link - there must also be at least one non-bigon face. Therefore,

$$
\left(e\left(\mathbb{G}_{A}\right)-e\left(\mathbb{G}_{A}^{\prime}\right)\right)-f\left(\mathbb{G}_{A}\right) \leq-1 .
$$


Furthermore, we have seen above that $\chi\left(\mathbb{G}_{A}^{\prime}\right) \geq 0$, hence

$$
2 g(D)=2-\chi\left(\mathbb{G}_{A}^{\prime}\right)+\left(e\left(\mathbb{G}_{A}\right)-e\left(\mathbb{G}_{A}^{\prime}\right)-f\left(\mathbb{G}_{A}\right)\right) \leq 2-0-1 .
$$

Since $g(D)$ is a non-negative integer, we conclude that $g(D)=0$.

This in turn, implies that the diagram $D$ is alternating; see Corollary 4.6 of [8]. Thus $D$ is a prime, alternating diagram that represents a Seifert fibered link. Now the work of Menasco [24] implies that $D$ is the standard diagram of a $(2, q)$ torus link.

Theorem 1.1 follows immediately by combining Theorem 5.10, Theorem 6.1, and Thurston's hyperbolization theorem for link complements [30]. Now we finish the proofs of Corollaries 1.2 and 1.3.

Proof of Corollary 1.2. Without loss of generality, assume $r_{j} \geq 3$ for all $j$. Then the diagram $D_{b}$ is a prime, $A$-adequate diagram and the corresponding state graph $\mathbb{G}_{A}$ contains no 2 -edge loops at all. Thus Theorem 1.1 implies that $K$ is hyperbolic.

Proof of Corollary 1.3. In [13, Corollary 3.21], we show that for a non-split, prime link $K$, any semi-adequate diagram $D(K)$ without nugatory crossings must be prime.

Conversely, if $D(K)$ is prime and semi-adequate, then Theorem 1.1 implies $K$ is hyperbolic or a $(2, q)$ torus link. Hence the link must also be prime.

\section{Acknowledgements}

During the course of this project, Futer was supported in part by NSF grants DMS-1007221 and DMS-1408682. Kalfagianni was supported in part by NSF grants DMS-1105843 and DMS-1404754. Purcell was supported in part by NSF grants DMS-1007437, DMS-1252687, and a Sloan Research Fellowship.

\section{References}

[1] Colin C. Adams, Augmented alternating link complements are hyperbolic. Low-dimensional topology and Kleinian groups (Coventry/Durham, 1984), London Math. Soc. Lecture Note Ser., Vol. 112, Cambridge Univ. Press, Cambridge, 1986, pp. 115-130. 
[2] Colin C. Adams, Toroidally alternating knots and links. Topology, 33 (1994), no. 2, 353-369.

[3] Colin C. Adams, Hyperbolic knots. Handbook of knot theory, Elsevier B. V., Amsterdam, 2005, pp. 1-18.

[4] Ian Agol, Lower bounds on volumes of hyperbolic Haken 3-manifolds. arXiv:math/9906182.

[5] Riccardo Benedetti and Carlo Petronio, Branched standard spines of 3-manifolds. Lecture Notes in Mathematics, Vol. 1653, Springer-Verlag, Berlin, 1997.

[6] Francis Bonahon and Laurent Siebenmann, New Geometric Splittings of Classical Knots, and the Classification and Symmetries of Arborescent Knots. Geometry and Topology Monographs, to appear, http://wwwbcf.usc. edu/ fbonahon/Research/Preprints/Preprints.html.

[7] Peter R. Cromwell, Knots and links. Cambridge University Press, Cambridge, 2004.

[8] Oliver T. Dasbach, David Futer, Efstratia Kalfagianni, Xiao-Song Lin and Neal W. Stoltzfus, The Jones polynomial and graphs on surfaces. Journal of Combinatorial Theory Ser. B, 98 (2008), no. 2, 384-399.

[9] David Futer and François Guéritaud, Angled decompositions of arborescent link complements. Proc. Lond. Math. Soc. (3), 98 (2009), no. 2, 325-364.

[10] David Futer, Efstratia Kalfagianni and Jessica S. Purcell, Dehn filling, volume, and the Jones polynomial. J. Differential Geom., 78 (2008), no. 3, 429-464.

[11] David Futer, Efstratia Kalfagianni and Jessica S. Purcell, Symmetric links and Conway sums: volume and Jones polynomial. Math. Res. Lett., 16 (2009), no. 2, 233-253.

[12] David Futer, Efstratia Kalfagianni and Jessica S. Purcell, Cusp areas of Farey manifolds and applications to knot theory. Int. Math. Res. Not. IMRN, 2010 (2010), no. 23, 4434-4497.

[13] David Futer, Efstratia Kalfagianni and Jessica S. Purcell, Guts of surfaces and the colored Jones polynomial. Lecture Notes in Mathematics, Vol. 2069, Springer, Heidelberg, 2013. 
[14] David Futer, Efstratia Kalfagianni and Jessica S. Purcell, Jones polynomials, volume, and essential knot surfaces: a survey. Knots in Poland III, Part I, Banach Center Publ., Vol. 100, Polish Acad. Sci. Inst. Math., Warsaw, 2014, arXiv:1110.6388, pp. 51-77.

[15] David Futer, Efstratia Kalfagianni and Jessica S. Purcell, Quasifuchsian state surfaces. Trans. Amer. Math. Soc., 366 (2014), no. 8, 4323-4343.

[16] David Futer and Jessica S. Purcell, Links with no exceptional surgeries. Comment. Math. Helv., 82 (2007), no. 3, 629-664.

[17] Adam Giambrone, Combinatorics of link diagrams and volume. J. Knot Theory Ramifications, 25 (2015), no. 1, 1550001, 21 pp.

[18] Michael Gromov, Volume and bounded cohomology. Inst. Hautes Études Sci. Publ. Math. (1982), no. 56, 5-99 (1983).

[19] Allen Hatcher, Notes on basic 3-manifold topology. http://www.math. cornell . edu/ hatcher/3M/3Mdownloads.html.

[20] Louis H. Kauffman, State models and the Jones polynomial. Topology, 26 (1987), no. 3, 395-407.

[21] Thilo Kuessner, Guts of surfaces in punctured-torus bundles. Arch. Math. (Basel), 86 (2006), no. 2, 176-184.

[22] Marc Lackenby, The volume of hyperbolic alternating link complements. Proc. London Math. Soc. (3), 88 (2004), no. 1, 204-224, with an appendix by Ian Agol and Dylan Thurston.

[23] W. B. Raymond Lickorish and Morwen B. Thistlethwaite, Some links with nontrivial polynomials and their crossing-numbers. Comment. Math. Helv., 63 (1988), no. 4, 527-539.

[24] William W. Menasco, Closed incompressible surfaces in alternating knot and link complements. Topology, 23 (1984), no. 1, 37-44.

[25] Makoto Ozawa, Closed incompressible surfaces in the complements of positive knots. Comment. Math. Helv., 77 (2002), no. 2, 235-243.

[26] Makoto Ozawa, Essential state surfaces for knots and links. J. Aust. Math. Soc., 91 (2011), no. 3, 391-404.

[27] Jessica S. Purcell, Hyperbolic geometry of multiply twisted knots. Comm. Anal. Geom., 18 (2010), no. 1, 101-120. 
[28] Jessica S. Purcell, On multiply twisted knots that are Seifert fibered or toroidal. Comm. Anal. Geom., 18 (2010), no. 2, 219-256.

[29] Morwen B. Thistlethwaite, On the Kauffman polynomial of an adequate link. Invent. Math., 93 (1988), no. 2, 285-296.

[30] William P. Thurston, Three-dimensional manifolds, Kleinian groups and hyperbolic geometry. Bull. Amer. Math. Soc. (N.S.), 6 (1982), no. 3, 357-381.

[31] Vladimir G. Turaev, A simple proof of the Murasugi and Kauffman theorems on alternating links. Enseign. Math. (2), 33 (1987), no. 3-4, $203-225$.

Department of Mathematics, Temple University

Philadelphia, PA 19122, USA

E-mail address: dfuter@temple.edu

Department of Mathematics, Michigan State University

EAST LANSInG, MI 48824, USA

E-mail address: kalfagia@math.msu.edu

Department of Mathematics, Brigham Young University

Provo, UT 84602, USA

E-mail address: jpurcell@math. byu.edu

Received February 24, 2014 